\title{
ПРИМЕНЕНИЕ ТЕОРИИ УСТОЙЧИВЫХ ПАРОСОЧЕТАНИЙ ДЛЯ АВТОМАТИЗАЦИИ ВЫБОРА ОБРАЗОВАТЕЛЬНОЙ ТРАЕКТОРИИ В ЭЛЕКТРОННОМ ОБУЧЕНИИ
}

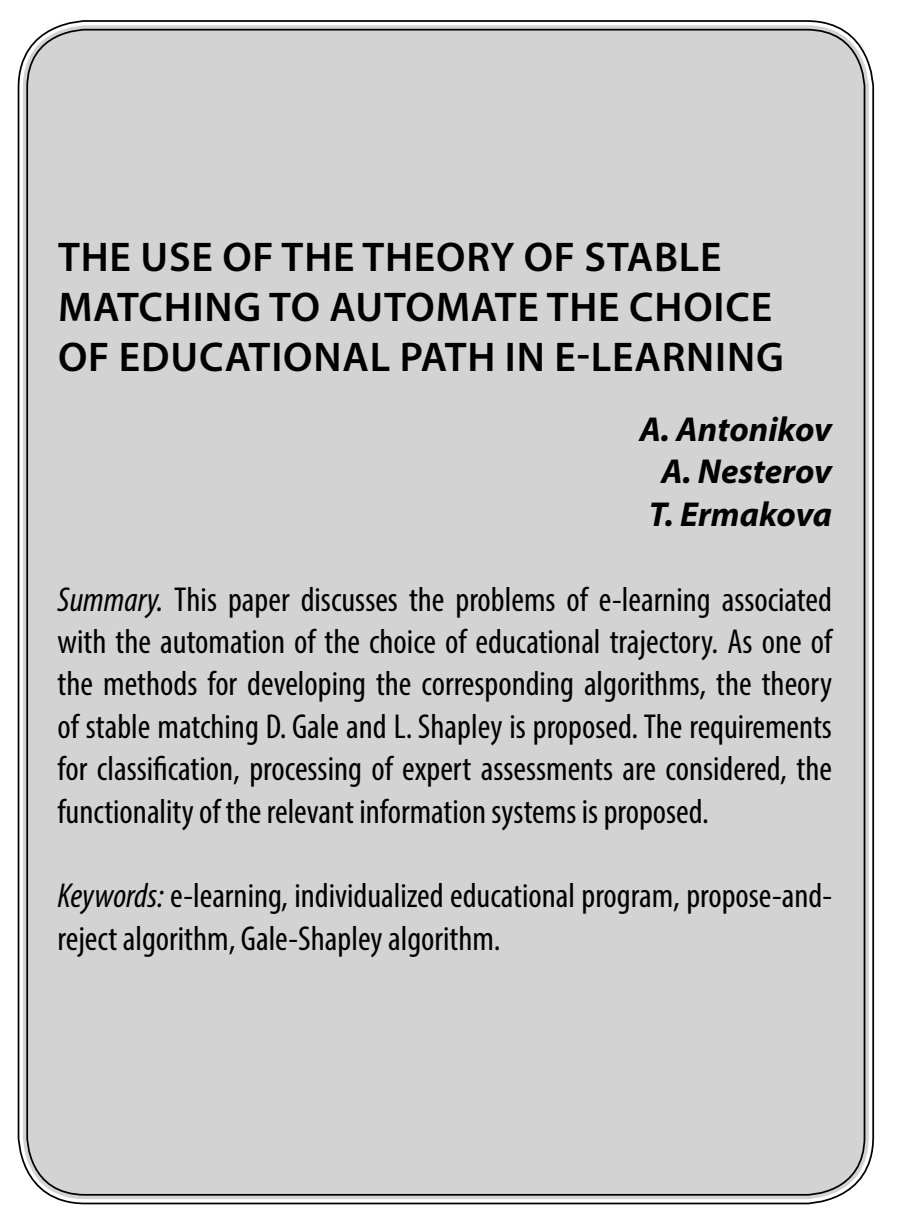

\section{Введение}

$\mathbf{K}$ онцепция электронного обучения описывает различные формы применения информационных технологий в образовательном процессе, начиная от распространения учебных материалов до проверки знаний в режиме «онлайн». Быстрое развитие информационных технологий постоянно приводит к появлению новых форматов электронного обучения. Одним из перспективных трендов последних лет в электронном образовании можно назвать применение индивидуальных образовательных траекторий в процессе получения знаний обучающимися. Данный подход предполагает наличие систем планируемых результатов обучения, диагностики уровня актуального развития обучающихся и прогнозирования ближайшей перспективы развития обучающихся [1].

\author{
Антоников Александр Александрович \\ Аспирант, ГАОУ ВО г. Москвы «Московский городской \\ педагогический университет» \\ antonikov.alexandr@gmail.com \\ Нестеров Андрей Владимирович \\ Д.ф.-м.н., профессор, ГАОУ ВО г. Москвы «Московский \\ городской педагогический университет» \\ andrenesterov@yandex.ru \\ Ермакова Татьяна Николаевна \\ К.т.н., дочент, ГАОУ ВО г. Москвы «Московский \\ городской педагогический университет» \\ ermaktat@bk.ru
}

Аннотация. В настоящей работе рассматриваются проблемы электронного обучения, связанные с автоматизацией выбора образовательной траектории. В качестве одного из методов для разработки соответствующих алгоритмов предлагается теория обобщенных паросочетаний Д. Гейла и Л. Шепли. Рассматриваются требования к классификации, обработке экспертных оценок, предлагается функционал соответствующих информационных систем.

Ключевые слова: дистанционное образование, индивидуальная образовательная траектория, задача об устойчивом паросочетании, алгоритм Гейла-Шепли.

\section{1. П^анируемые результаты обучения}

Образовательная программа по направлению подготовки формируется образовательной организацией самостоятельно на основе утвержденных локальных нормативных актов этой организации, не противоречащих ФГОС ВО по данному направлению подготовки. Номера семестров, а также трудоемкость и форма промежуточной аттестации каждой дисциплины, модуля или практики, входящих в обязательную часть, устанавливаются образовательной организацией самостоятельно и фиксируются в рабочей программе дисциплины, при этом в рабочей программе указывается место дисциплины в образовательной программе и перечень дисциплин, формирующих базовые знания для успешного освоения программы. В соответствии с этими нормативными документами профессорско-преподавательский состав 


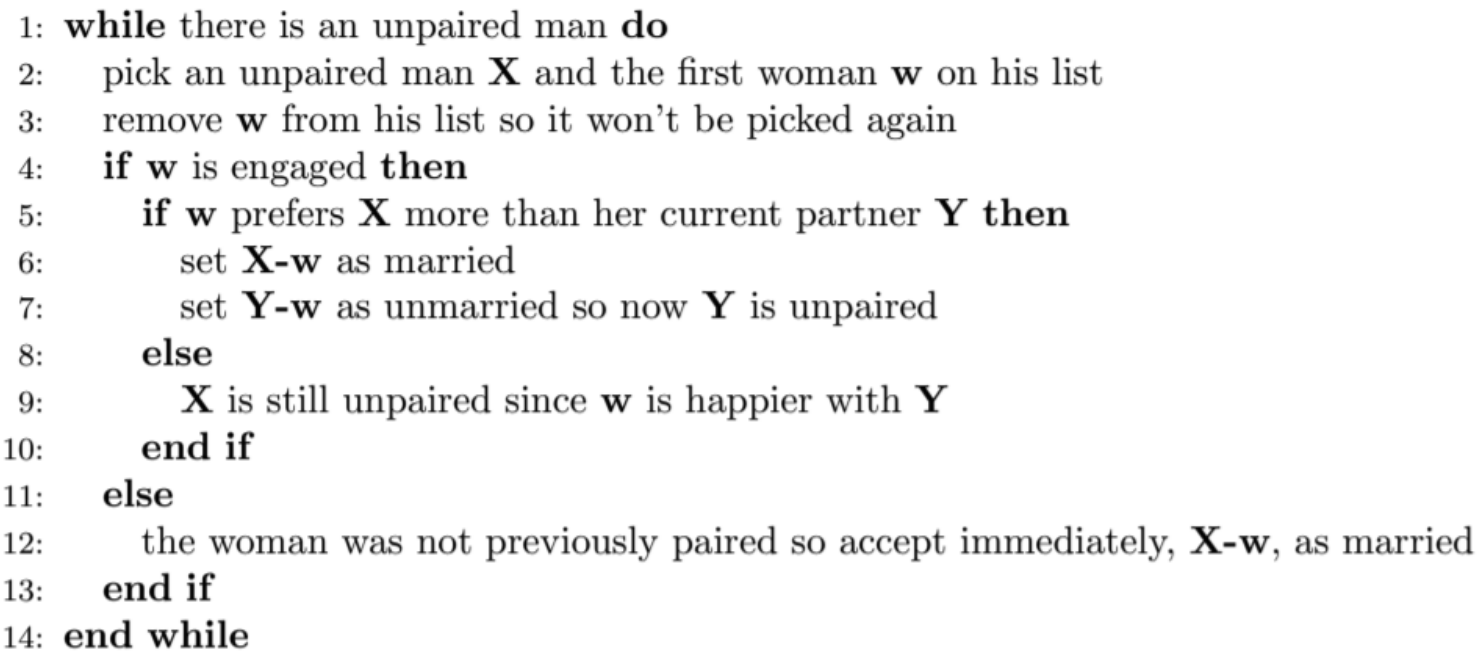

Рис. 1. Классический алгоритм Гейла-Шепли

формирует перечень заданий, которые обучающийся должен выполнить в процессе освоения дисциплины в рамках практических и самостоятельных занятий, при этом набор заданий должен максимально способствовать освоению обучающимся требований, установленных в результатах освоения дисциплины [2-4].

\section{2. ПоАбор за аний $\triangle \wedge$ Я обучаюшихся с учетом$$
\text { степени освоения материала }
$$

Так как уровень усвоения материалов дисциплины у разных обучающихся может значительно варьироваться, задачу распределения заданий обучающимся в терминологии теории графов можно представить как задачу поиска паросочетания максимальной мощности в двудольном графе (Д. Кенига, Ф. Холла) [5]. Эта задача позволяет учитывать предпочтения относительно возможных пар, которые связаны с разными группами объектов, представляемых в теоретико-игровом смысле в качестве игроков. Теория обобщенных паросочетаний, предложенная Д. Гейлом и Л. Шепли [6] в 1962 году, позволяет учитывать предпочтения отдельных игроков при выборе распределения. Под введенным этими учеными термином «устойчивое обобщенное паросочетание» понимается паросочетание, от которого игроки не захотят отказаться. Данная теория доказывает, что устойчивое паросочетание существует постоянно. Также учеными был предложен механизм построения устойчивого паросочетания. В отличие от аналогичной задачи поиска паросочетания в графе в классическом варианте ими была использована модель «один ко многим». В этой модели игроки одной из подгрупп могут входить в состав не одной, а нескольких пар. На рисунке 1 представлен классический алгоритм Гейла-Шепли. В дальнейшем в исследованиях Д. Гейла, Э. Рота, Л. Дубинса, Д. Фридмана, М. Сотомайора,
Р. Ирвинга, Ч. Блэра, Д. Хатфильда, П. Милгра [7] и других ученых данная задача рассматривается с других сторон и предлагаются разные подходы к процессу моделирования предпочтений и определения устойчивости. В теории обобщенных паросочетаний для обозначения групп игроков в моделях «один ко многим» используются термины «абитуриенты» и «вузы». Стоит отметить, что модели являются по своей сути абстрактными, а результаты, полученные с их помощью, могут быть использованы применительно к любым подобным системам.

В работе Д. Гейла и Л. Шепли, а также во многих исследованиях А.Рота и М.Сотомайора предполагается, что предпочтения, имеющиеся у игроков, заданы линейно, т.е. каждый игрок может упорядочить потенциальных партнеров строго по уровню их предпочтительности. В реальности предпочтения игрока часто основываются на недостаточно точных измерениях, также может не хватать информации. В таком случае становится актуальной задача изучения моделей, в которых некоторые альтернативы могут не отличаться для игрока по предпочтительности, т.е. предпочтения игрока не могут быть представлены линейно. Существует случай, когда отношения предпочтения игроков $P_{i}$ являются слабыми порядками, и тогда возникает проблема построения устойчивого обобщенного паросочетания. Эта проблема впервые была выявлена А. Ротом, в дальнейшем над ней работал Р. Ирвинг, который предложил модифицированное определение устойчивого паросочетания. Также эта проблема была рассмотрена в трудах А. Аблукадироглу, Т. Сонмеза, П. Патака, Д. Манлова, А. Эрджила и Х. Эрдила в 2003-2009 гг. [7].

При этом необходимо учесть, что результат поиска устойчивых паросочетаний может быть значительно искажен в случае предоставления некорректной информации по предпочтениям игроков. 


\section{3. К^ассификачия заАаний $\triangle \wedge$ Автоматизированного распределения заланий}

Применительно к задаче распределения задач обучающимся необходимо максимально точно классифицировать сильные и слабые стороны, продемонстрированные обучающимся в процессе решения задач и получения образовательных результатов решения заданий [8-10].

Основой для данной классификации должна быть информация о заданиях и результатах выполнения заданий, полученная от экспертов, обработанная затем с помощью ранговых процедур. Классификация задач должна учитывать:

1. Возможное наличие у задач межпредметного взаимодействия, которое будет говорить о том, что для успешного решения задачи нужно применить навыки и знания, полученные при изучении другой дисциплины;

2. Разбиение итоговой оценки на ряд критериев, каждый из которых может иметь свою шкалу оценки;

3. Список формируемых компетенций, обозначенных в образовательной программе и рабочем плане дисциплины;

4. С учетом временного ограничения на решение заданий представляется важным также получить экспертные оценки на длительность выполнения заданий обучающимися.

Результатом обработки мнений экспертов будет являться база классифицированных заданий, из которой задания можно распределять обучающимся, основываясь на результатах оценки выполненных ранее заданий и с учетом того, что обучающийся за время, отведенное на выполнение заданий, должен максимально охватить все темы дисциплины и сформировать максимальное количество компетенций.

Успешность выполнения задания, в свою очередь, должна проверяться с учетом соответствия овладения обучающимся материала по различным критериям данной классификации. Набор заданий, выполняемых обучающимся в рамках изученной дисциплины, должен затрагивать все темы, обозначенные в рабочем планам дисциплины, и формировать у обучающегося перечисленные там же компетенции.

\section{4. Функциональные требования \\ к информационной системе \\ распремеления заланий \\ среАи обучаюшихся}

Процесс обработки мнения экспертов и распределения заданий должен быть автоматизирован. Для этого нужно разработать программный продукт, автоматизирующий выполнение следующих функций:

1. Добавление пользователей с распределением прав по ролям (студент, преподаватель-эксперт)

2. Добавление дисциплины с указанием списка формируемых компетенций и списка ранее изученных предметов, на которых базируется данная дисциплина, списка изучаемых тем, количества часов, выделенных на изучение дисциплины, в том числе с указанием часов, отведенных на практические и лабораторные занятия, а также на самостоятельную работу;

3. Добавление задания, где указывается дисциплина/список дисциплин, тема/список тем, наименование задачи, краткая постановка, присутствует возможность приложить дополнительные материалы для решения задачи в виде файлов;

4. Создание анкет/наборов анкет для опроса мнения экспертов о задачах;

5. Проведение оценки задания с помощью опроса эксперта по анкете;

6. Обработка мнений экспертов с целью получения объективной классификации задачи;

7. Проверка списка задач, оцененных экспертами по дисциплине на полноту охвата изучаемых тем, формируемых компетенций, количества часов, отведенных на практические занятия;

8. Назначение обучающемуся задания, основанное на подборе паросочетания с учетом предпочтений, заключающихся в качественном освоении дисциплины, с учетом оценки текущего уровня знаний, основанном на результатах выполнения заданий, и с учетом результатов оценки заданий по базовым дисциплинам, изученным ранее;

9. Прикрепление к заданию результата выполнения в виде файлов/наборов файлов;

10. Оценка задания преподавателем по критериям, выделенным экспертами в процессе классификации задачи, с возможностью оставления комментария к результатам;

11. Для обучающегося должна быть возможность просмотреть все ранее выполненные задания, с оценками, комментариями, вложениями;

12. Для преподавателей должна быть возможность просмотреть список выполненных обучающимися заданий, нуждающихся в оценке;

13. Для преподавателей должна быть возможность просмотреть информацию о степени освоения обучающимся дисциплины, полученной на основе данных о выполненных по дисциплине заданий.

Часть описанных функций в том или ином виде присутствует в используемых в образовательном учрежде- 


\section{Система дистанционного образования}

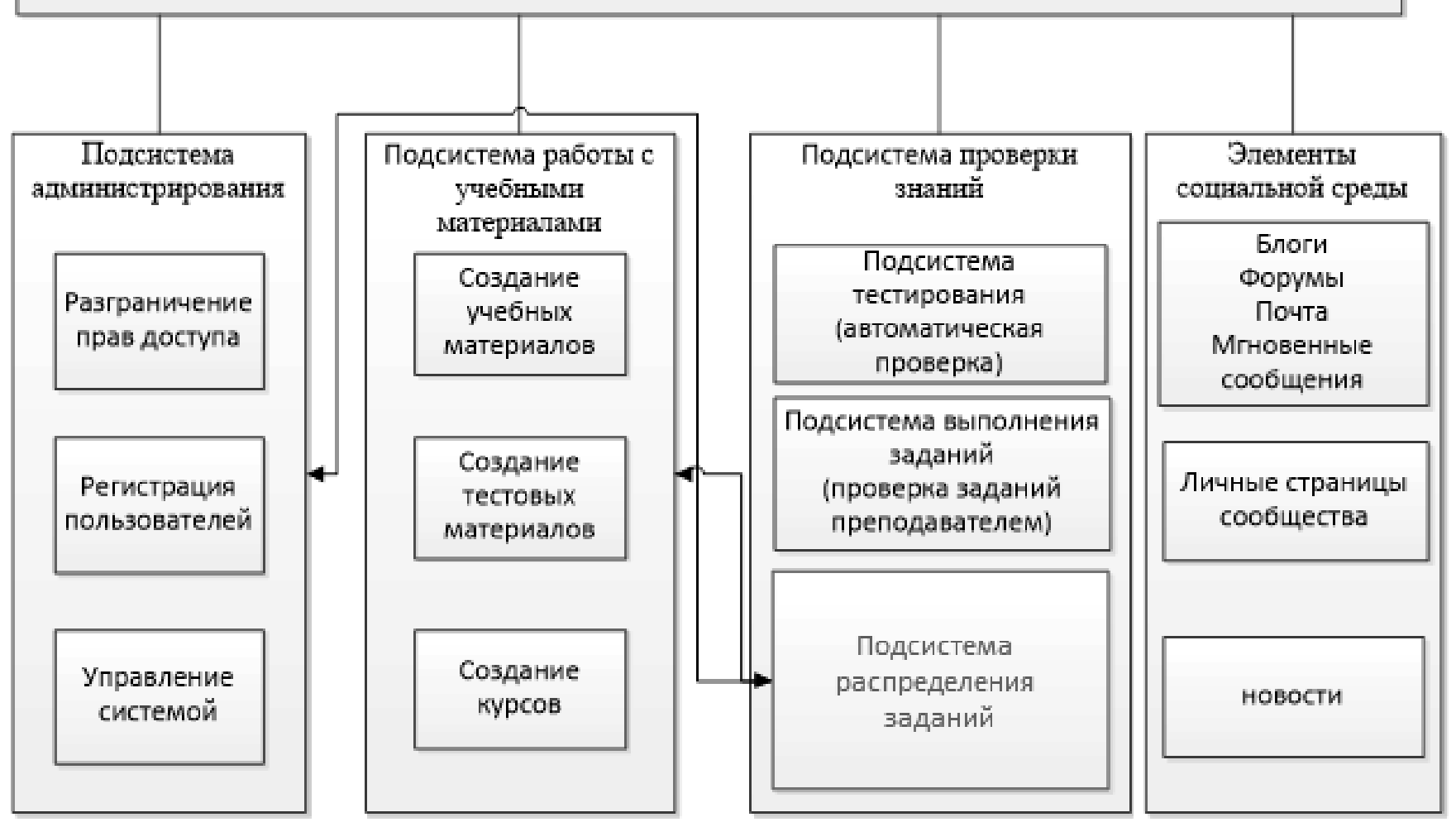

Рис. 2. Место модуля в системе дистанционного образования

нии информационных системах дистанционного образования (СДО), и имеет смысл рассматривать данные требования как требования к реализации нового обособленного функционала подсистемы проверки знаний СДО (рисунок 2).

Реализация данного модуля в значительной степени будет определяться возможностями СДО и потребностями образовательной организации [11]. При проектировании и реализации подобной подсистемы необходимо реализовывать функционал максимально отделяемыми атомарными единицами для обеспечения возможности вносить последующие корректирую- щие изменения, не затрагивая остальной функционал сдО.

\section{Зак^ючение}

Исходя из того, что во ФГОС зафиксирована возможность применения индивидуальной образовательной траектории в процессе обучения, появление в системах электронного обучения соответствующего функционала лишь вопрос времени, а поднятые в статье вопросы классификации заданий для последующего распределения среди обучающихся будут актуальны для разработчиков подобных решений.

\section{ЛИТЕРАТУРА}

1. Барсукова К.Н., Чискидов С. В., Павличева Е. Н. Актуальные проблемы автоматизации учета результатов обучения студентов в вузе (на примере ИМИИЕН ГБОУ ВПо МГПУ) // Информационные ресурсы России.—2015.— № 3 (145).—C. 37-39.

2. Gorelov G. V., Romashkova 0. N. Influence of Russian, Spanish and Vietnamese speech characteristics on digital information transmission quality // IEEE International Symposium on Industrial Electronics Proceedings of the IEEE International Symposium on Industrial Electronics, ISIE'96. Part 1 (of 2). sponsors: IEEE, Warsaw University of Technology. Warsaw, Poland, 1996, p.311-313.

3. Bobrikova E., Gaidamaka Y., Romashkova 0. The application of a fluid-based model for the analysis of the distribution time of a file among users in peer-to-peer network // Selected Papers of the II International Scientific Conference "Convergent Cognitive Information Technologies" (Convergent 2017). CEUR Workshop Proceedings, Volume 2064. p. 55-61. Available at: http://ceur-ws.org/Vol-2064/paper06.pdf. 
4. Gudkova, I.A., Romashkova, 0.N., Samoylov, V. E. Determination of the range of the guaranteed radio communication in wireless telecommunication networks of IEEE802.11 standard with the use of ping program // B сборнике: CEURWorkshop Proceedings 8. Cep. "ITTMM 2018 — Proceedings of the Selected Papers of the 8th International Conference "Information and Telecommunication Technologies and Mathematical Modeling of High-Tech Systems"'" 2018. C. 54-59.

5. Оре 0. Графы и их применение.-М.: Изд-во «Мир», 1965.

6. Gale D., Shapley L. S. College admissions and the stability of marriage // American Mathematical Monthly. — 1962.—Vol. 69.—- P. 9-16.

7. Алескеров Ф.Т., Хабина Э. Л., Шварц Д. А. Бинарные отношения, графы и коллективные решения.—- М.: Физматлит, 2012.

8. Ромашкова 0.Н., Ермакова Т. Н. Этапы реализации методики выбора информационной модели для оценки показателей качества обучения // В сборнике: Междисциплинарные исследования в области математического моделирования и информатики Материалы 7-й научно-практической internet-конференции. отв. ред. Ю.С. Нагорнов. 2016. С. 318-321.

9. Ермакова Т.Н., Ромашкова 0.Н., Пономарева Л. А. Модернизированная структура управления образовательной системой // Вестник Брянского государственного технического университета. 2019. № 6 (79). С. 84-91.

10. Ромашкова 0.Н., Ермакова Т. Н. Применение инфокоммуникационных технологий для анализа показателей качества обучения образовательного комплекса // В сборнике: Технологии информационного общества Х Международная отраслевая научно-техническая конференция: сборник трудов. 2016. C. 388-389.

11. Антоников А.А., Чискидов С. В., Павличева Е. Н. Разработка модуля системы дистанционного обучения для проверки знаний в области программирования // Информационные ресурсы России. 2012. № 3 (127). С. 32-34.

(c) Антоников Александр Александрович ( antonikov.alexandr@gmail.com ),

Нестеров Андрей Владимирович ( andrenesterov@yandex.ru ), Ермакова Татьяна Николаевна ( ermaktat@bk.ru ).

Журнал «Современная наука: актуальные проблемы теории и практики»

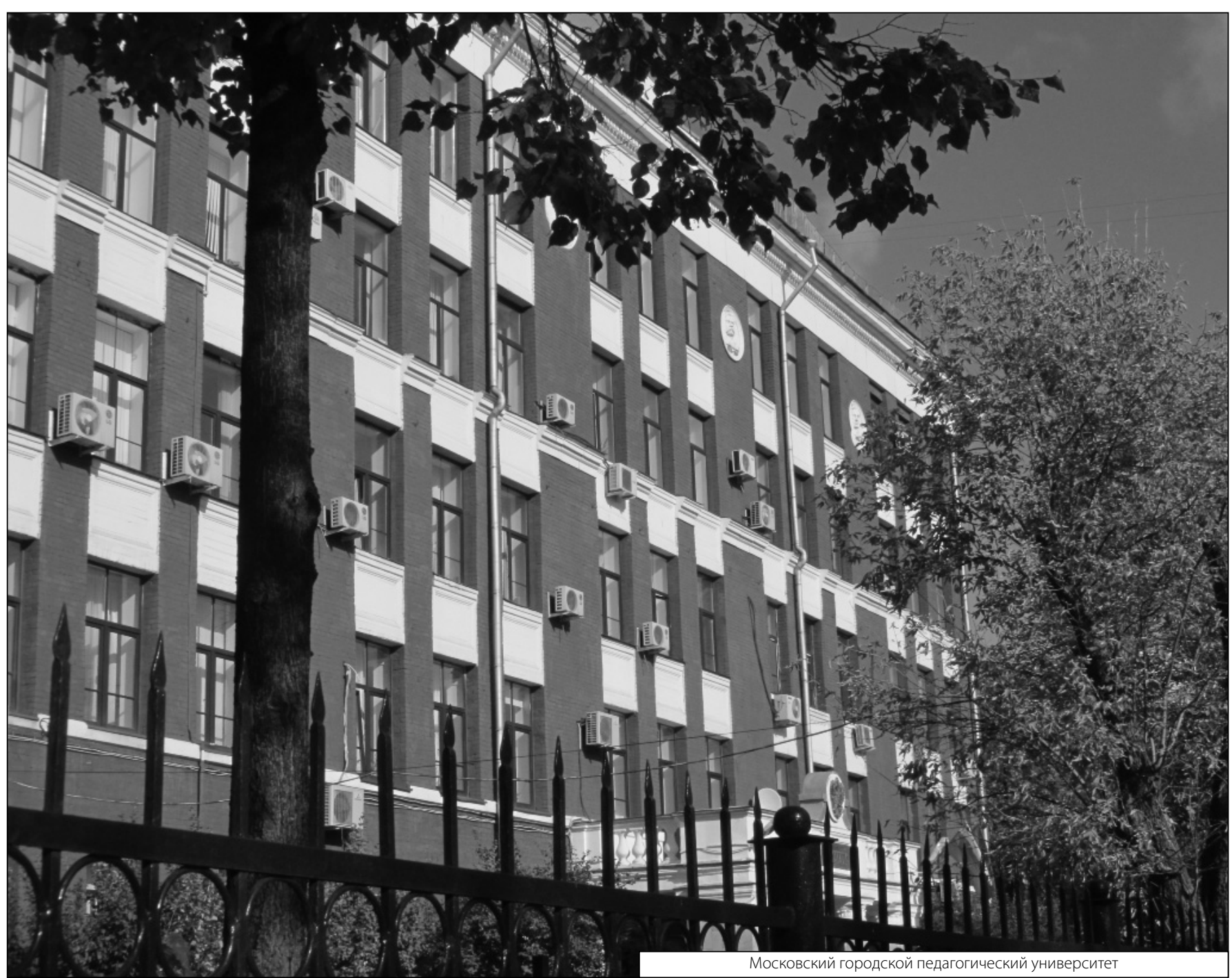

